\title{
CÓMO LEER Y GENERAR PUBLICACIONES CIENTÍFICAS. REVISIÓN CRIITICA DEL ARTÍCULO "Inhaled budesonide in the treatment of early COVID-19 (STOIC): a phase 2, open-label, randomised controlled trial", de Sanjay Ramakrishnan y cols. PUBLICADO EN LANCET RESPIR MED 2021; 9: 763-72 \\ CRITICAL REVIEW OF THE ARTICLE Inhaled budesonide in the treatment of early COVID-19 (STOIC): a phase 2, open-label, randomized controlled trial", of Sanjay Ramakrishnan y cols. PUBLISHED ON LANCET RESPIR MED 2021; 9: 763-72
}

Klgo. Claudio Olmos 1,2,a, Dr. Ernesto Guiraldes ${ }^{2, b}$

1. Unidad de Investigación, Clínica INDISA.

2. Escuela de Medicina, Universidad Andrés Bello.

a. Kinesiólogo, Magister en Salud Pública, Doctor en Metodología de Investigación Biomédica y Salud Pública.

b. Médico, Pediatra, Diplomado en Educación Médica.

\section{INTRODUCCIÓN}

Probablemente todos quienes nos dedicamos al ámbito de la salud, estamos de acuerdo en que resulta fundamental incorporar la evidencia científica a nuestro quehacer. La Medicina Basada en la Evidencia (MBE), entendida como la utilización consciente, explícita y juiciosa de la mejor evidencia clínica disponible para tomar decisiones sobre el cuidado de los pacientes individuales (1), se ha vuelto un imperativo en el ejercicio de la profesión. Sin embargo, detrás de esa obligación, existe la dificultad que dice relación con nuestra capacidad para evaluar la evidencia disponible, en su mérito, contexto y pertinencia. Se dice que una buena evaluación crítica no se fundamenta sólo en identificar y citar aquellas debilidades y limitaciones de las que adolece, sino, también, evaluar su impacto en la totalidad del estudio. Aquello requiere de competencias asociadas a la metodología de investigación, pero también práctica y permanente discusión académica con nuestros pares. Bien lo decía el Dr. Manuel Bobenrieth cuando explicaba que, debido a que la práctica basada en la investigación depende de los hallazgos de estudios científicos publicados, cada estudio de investigación debe ser evaluado críticamente, no solamente para identificar argumentos en favor de su validez, sino también su importancia, su novedad y su proyección e influencia en la comunidad (2). En pos de lo anterior, es importante desmitificar ciertas creencias que circulan en torno a la publicación científica y que claramente resultan contradictorios con la realidad objetiva. Por ejemplo, un artículo publicado en una revista de prestigio, con autores reconocidos por su prác- tica profesional o publicaciones anteriores, no son garantía de su validez (3)

En esta oportunidad, hemos de analizar un artículo publicado recientemente en la revista Lancet (en su edición de Medicina respiratoria), titulado "Inhaled budesonide in the treatment of early COVID-19 (STOIC): a phase 2, open-label, randomised controlled trial'(4). Se aconseja leer esta revisión teniendo el artículo en cuestión, a mano.

\section{REVISIÓN CRÍTICA}

\section{... del Título y los Autores}

El título es conciso, fácil de entender y claramente indicativo del contenido del estudio. Eventualmente podría causar complicaciones la sigla STOIC (STerOids in COVID-19 Study), razón por la cual, no se aconseja usar abreviaturas en los títulos. Respecto a los autores, están correctamente descritos en cuanto sus nombres y filiación, y se señala que los dos primeros contribuyeron en partes iguales. De los numerosos colaboradores no se especifica su rol, cuestión que no es una obligación en la publicación, pero sí lo es al momento de enviar el trabajo a una revista. Por ende, nos queda confiar en que los editores hayan considerado que se justificaba tal cantidad de participantes.

\section{... del Resumen}

Se trata de un resumen inusualmente largo. La mayoría de las veces se solicita un resumen de 250 palabras. En esta ocasión se presenta uno de 616 palabras. Esto no favorece el entendimiento del trabajo, ya que desvía la atención de lo que realmente importa en el trabajo. Es ahí donde aparece tal vez, el primer cuestionamiento: por qué los autores no fueron capaces de sintetizar sus resultados. Lo otro que llama la atención de este apartado, es que, en el primer párrafo, los autores se aventuran a hipotetizar que el uso de corticoides inhalados podría ser un tratamiento efectivo contra el COVID-19. Si bien, la hipótesis es parte fundamental del método científico, los investigadores deben mantener la neutralidad en la forma y en el fondo; iniciar su investigación a partir de una duda razonable (equipoise). De hecho, por eso el análisis estadístico inferencial se realiza sobre la base de asumir la hipótesis nula como verdadera. Al poner su hipótesis en el primer párrafo, se instaura inmediatamente la intencionalidad de los autores

\section{... de la Introducción}

(Estado del arte, Problema y Objetivos). En términos generales, la introducción presenta el tema adecuadamente, resaltando la relevancia del tema e indirectamente, justificando la importancia de estudiar alternativas de tratamiento para la enfermedad. Lo hace con un adecuado número de referencias y muy actualizada. Esta sección incorpora algunos elementos que pudiesen hacer pensar en la plausibilidad biológica de la hipótesis; sin embargo, el problema de investigación propiamente tal, no se plantea. En ese sentido, el trabajo parece dar por sentado que literalmente todo el mundo, asume como un problema, la falta de tratamiento de la COVID-19, y por eso, aun cuando no lo explicite, el lector puede imaginarse cuál

Autor para correspondencia:

Claudio Olmos González

claudioolmos@gmail.com 
es la pregunta de investigación. Aún así, el problema debiera estar explícito. Por el contrario, el objetivo se plantea adecuadamente en el último párrafo de la introducción, como es debido.

\section{...del Método}

Como es de imaginarse, esta sección es la que está expuesta a los más fuertes cuestionamientos en todo estudio. Y es que la forma en que se diseña el trabajo, se selecciona la muestra, así como la modalidad de medir las variables, esconden la mayor cantidad de sesgos, que repercuten directamente en la validez de los resultados. Se presenta un ensayo clínico aleatorizado, controlado, sin ciego, realizado en la comunidad. Este diseño parece apropiado para comprobar la efectividad de un tratamiento, aun cuando los pacientes sepan el tratamiento que están recibiendo. Lo óptimo sería un doble ciego, de manera que tanto pacientes como investigadores no sepan quien está en el grupo de intervención y quien, en el control, evitándose así el efecto placebo; sin embargo, construir un inhalador placebo, probablemente requiere una logística que la premura de encontrar posibles tratamientos, no permite. No es allí, donde el estudio presenta su principal falencia. Se reclutaron adultos con sintomatología COVID, que no hubieran usado corticoides inhalados o sistémicos en los últimos 7 días, los que fueron asignados a dos grupos. A un grupo se le dio terapia con budesonida en inhalador de polvo seco y al otro grupo, las recomendaciones estándar (antipiréticos en caso de fiebre, antiinflamatorios no esteroidales y miel para la tos). Ambos grupos recibieron la visita de una enfermera al día 0, 7 y 14. Los sujetos de estudio debían registrar su oximetría, temperatura y síntomas diarios, todo ello autorreportado. A los sujetos pertenecientes al grupo de corticoides, se les pidió que dejaran de usar inhalador cuando ellos mismos consideraran que se habían recuperado. Lo anterior trae consigo el gran dilema de que la veracidad de la información recae enteramente en los sujetos de estudio. A pesar de la reputada confiabilidad del servicio de salud británico y de la proverbial disciplina de sus usuarios...al lector crítico de este estudio no puede asegurársele de manera fidedigna que no hubo... automedicación en los sujetos de ambos grupos o que no hubo otras intervenciones más allá de las registradas por el sujeto. Además, aún cuando ninguna de las anteriores interferencias se haya presentado, lo registrado depende de la subjetividad de la persona. Difícilmente uno podría objetivar la efectividad de un tratamiento, a lo que el paciente determine como "recuperado". Este asunto tomará especial importancia, cuando se observen las diferencias en los resultados de uno y otro grupo.

En contraste, el apartado referente al análisis estadístico, particularmente en su presentación visual, con gráficos de gran factura técnica, goza de una robustez admirable, ante lo cual no es difícil que los lectores, probablemente impresionados, puedan pensar que los resultados analizados de esa manera, seguramente han de ser válidos. Hacemos especial énfasis en esto, pues parece prudente recordar que el análisis estadístico no es principal elemento que le confiere validez interna a un trabajo. De hecho, si hay algo en que contribuye un buen análisis inferencial, es en aportar validez externa ("qué tan extrapolables a la población general son los resultados"); sin embargo, la validez interna, estará determinada principalmente por el diseño y metodología de recolección de datos.

Al finalizar esta sección, el artículo no entra en mayores detalles sobre los aspectos éticos, pero sí se dice que el estudio fue financiado por el Instituto Nacional de Investigación en Salud (NIHR), el Centro de Investigación Biomédica y por AstraZeneca. Asimismo, se afirma que las anteriores entidades no tuvieron injerencia ni en el estudio ni en la decisión de publicarlo.

\section{... de los Resultados}

El resultado principal (outcome) se definió como visitas al servicio de urgencia relacionadas con COVID-19, incluyendo evaluaciones en sala de emergencias u hospitalización. Los resultados fueron analizados tanto "por protocolo" como "por intención de tratar". Esto último es importante porque permite mantener hasta el final el balance de los factores pronósticos conocidos y desconocidos entre ambos grupos, disminuyendo la probabilidad de sesgar los resultados. Sin embargo, llaman la atención algunos datos que en esta sección se revelan. La población definida como "non-white", está sub-representada con un $7 \%$ del total, cuestión que ya podría atentar contra la validez externa (extensión y forma en que los resultados de un estudio pueden ser generalizados a diferentes sujetos, poblaciones, etc.). En esas condiciones, cuando se analizó la población según protocolo, el resultado principal ocurrió en $10(14 \%)$ participantes en el grupo de atención habitual y en $1(1 \%)$ participante en el grupo de budesonida, lo que indica una reducción del riesgo relativo del $91 \%$ para la budesonida. Es decir, en una muestra de 146 participantes, 11 personas visitaron el servicio de urgencia: la conclusión que sustenta la reducción de riesgo del $91 \%$ (estadísticamente significativa, por cierto), se desprende de lo que ocurrió con 11 personas. Por otra parte, en referencia a manifestaciones clínicas en estudio, la temperatura más alta registrada en ambos grupos fue de $36,6^{\circ} \mathrm{C}$. Entonces, es justo pensar que la mayoría de los sujetos tuvo una enfermedad leve. En el diagrama de violín, se muestra una diferencia promedio de temperatura de 0,49 entre ambos grupos, con un intervalo de confianza de 0,32 a $0,66^{\circ} \mathrm{C}$, que es estadísticamente significativo ( $p<0,001)$. Es decir, el análisis estadístico nos está diciendo que la diferencia de medio grado de temperatura entre ambos grupos, siempre bajo $36,6^{\circ} \mathrm{C}$, no es producto del azar y, por ende, sería un efecto atribuible al corticoide inhalado. Aquí resulta oportuno recordar que esta conclusión, está basada en el autorreporte; cuestión que a lo menos podría considerarse como un sesgo de información, que atenta contra la validez interna del estudio. Además, cabe preguntarse, cuál es la relevancia clínica de este hallazgo, (en oposición a su significación estadística), si se está manifestando dentro un rango de temperatura normal.

\section{... de la Discusión}

Hasta este punto, el lector habrá notado que el trabajo adolece de algunos sesgos de selección, por el hecho de trabajar con una población que no es representativa de la población general; de algunos sesgos de información, principalmente por el poco control que tiene sobre el registro de información; y de algunos sesgos de confusión, al no considerar la posible automedicación o el momento epidémico en que se realizó el estudio. Uno esperaría que en la discusión se comentaran todos estos aspectos. Es cierto que resulta complejo controlar todos los sesgos, pero afortunadamente existe este eventual apartado donde los autores tienen la posibilidad de discutir sus resultados y las limitaciones. Sin embargo, en este aspecto, no se hace el ejercicio de la autocrítica. Los autores abren la discusión afirmando: "Hemos demostrado que la budesonida inhalada, administrada durante un período breve, podría ser un tratamiento eficaz del COVID-19 en etapa temprana en adultos. Este efecto, con una reducción relativa del $91 \%$ del deterioro clínico, es equivalente a la eficacia observada después del uso de las vacunas COVID-19 y mayor que la reportada en cualquier tratamiento utilizado en pacientes hospitalizados y pacientes con COVID-19 grave."

Más adelante prosiguen: "Además, en los países de ingresos altos, la budesonida inhalada podría funcionar como un complemento para reducir la presión sobre los sistemas de atención de la salud hasta que se pueda lograr una vacunación generalizada contra el SARS-CoV-2. Además, es poco probable que la eficacia de la budesonida inhalada se vea afectada por alguna variante emergente del SARS- 
CoV-2, que ha sido motivo de preocupación con la implementación de la vacuna." "...Existe la posibilidad de que el efecto inmunomodulador de los corticoides inhalados también pueda aplicarse a cualquier virus en futuras epidemias, pero esto requiere una mayor evaluación"

Los autores de este análisis crítico, consideramos que no es posible emitir conclusiones tan taxativas sobre la efectividad de la intervención, basadas en las 11 personas que debieron acudir a servicios de urgencias.

\section{CONCLUSIONES}

Toda investigación debe evaluarse en su mérito y de acuerdo al contexto en que esta fue redactada. Sobre todo, en materias sobre las que se sabe poco y/o cuando el comportamiento del fenómeno de interés evoluciona rápidamente, tal como ha sido la enfermedad provocada por el SARS-CoV-2. LoS artículos deben ser entendidos como información científica rigurosa. Cada estudio debiera entregarnos conclusiones basadas en una metodología válida y consistente. Cada vez que leemos un "paper", estamos recibiendo información nueva, debidamente respaldada. Por lo tanto, si el artículo pasa este filtro metodológico, debiéramos incorporarlo a nuestro quehacer. Por el contrario, si un trabajo no aporta nada nuevo o bien, lo que concluye no constituye un legítimo corolario del estudio realizado, estamos en nuestro derecho a desestimar lo que allíse propone. Por lo tanto, cada profesional de la salud, debiera ser capaz de ser agente activo de una permanente discusión académica.

LoS autores declaran no presentar conflicto de interés.
REFERÉNCIAS

1. Sackett DL, Rosenberg WMC, Gary JAM, Haynes RB, Richardson WS. Evidence based medicine: what it is and what itisn't. BMJ. 1996; 312:71-2.

2. Bobenrieth M. Normas para revisión de artículos originales en Ciencias de la Salud. Int I Clin Heal Psychol. 2002;2(3):509-23.

3. Bobenrieth M. Mitos y realidades en torno a la publicación cientifica. Med Clin (Barc). 2000;114(9):339-41.

4. Ramakrishnan S, Nicolau D, Langford B, Mahdi M, Jeffers $\mathrm{H}, \mathrm{Mw}$ wasuku C, et al. Inhaled budesonide in the treatment of early COVID-19 (STOIC): a phase 2, open-label, randomised controlled trial. Lancet Respir Med. 2021:9:763-72. 